\title{
Two user-friendly models and a simple cutoff value of neutrophil to lymphocyte ratio (NLR) for early diagnosis of diabetic nephropathy in middle-elderly aged patients with type 2 diabetes: A cross- sectional study
}

Yiming Tian ( $\sim$ Yiming_Tian@foxmail.com )

the First Hospital of Qinhuangdao https://orcid.org/0000-0002-6162-0671

Tao Li

the First Hospital of Qinhuangdao

Hongyuan Gu

the First Hospital of Qinhuangdao

Rui Wang

the First Hospital of Qinhuangdao

Chunming Ma

the First Hospital of Qinhuangdao

Rui Wang

the First Hospital of Qinhuangdao

Research article

Keywords: Albumin to creatinine ratio, cardiovascular disease, inflammation, diabetic nephropathies, diabetes mellitus, type 2

Posted Date: July 30th, 2020

DOI: https://doi.org/10.21203/rs.3.rs-24861/v2

License: (c) (i) This work is licensed under a Creative Commons Attribution 4.0 International License.

Read Full License 


\section{Abstract}

Background: Neutrophil to lymphocyte ratio (NLR) has been shown to predict worse outcomes of diabetic nephropathy (DN). This cross-sectional study aimed to investigate the association of NLR and DN in middle-elderly aged patients with type 2 diabetes, and attempted to confirm an optimized cutoff value of NLR for DN prediction.

Methods: A total of 146 patients with type 2 diabetes were retrospectively included in this study. DN was defined as urine albumin to creatinine ratio (UACR) $\geq 30 \mathrm{mg} / \mathrm{g}$, or effective glomerular filtration rate (eGFR) $\leq 60 \mathrm{ml} / \mathrm{min} \cdot 1.73 \mathrm{~m}^{2}$. To evaluate the predictive role of NLR, logistic regression analysis and receiver operating characteristics $(\mathrm{ROC})$ curve analysis were applied. Canonical discriminant functions were used to construct the discriminant equations.

Results: NLR, diabetes duration, systolic blood pressure (SBP) and lipo-protein a [Lp(a)] independently predicted DN diagnosis after adjusted by multi-variables. NLR value of 2.04 had a sensitivity of $48.9 \%$ and a specificity of $80.8 \%$ in predicting DN, with area under the curve (AUC) of 0.666 . When the threshold of NLR was elevated to 2.50 , the specificity and sensitivity were $90.9 \%$ and $29.8 \%$, respectively. Userfriendly model 1 and model 2 were constructed using the independent risk factors mentioned above, with the AUC of 0.819 and 0.817 , respectively.

Conclusions: Two models of user-friendly equations were constructed for early prediction of DN, which could be easily calculated and stored in office computer. NLR threshold of 2.50 is recommended in clinical use to identify the patients at high risk of DN, for its high specificity and remarkable convenience.

\section{Background}

Diabetic nephropathy (DN) occurs in $20-40 \%$ of patients with diabetes, it has become one of the leading causes of end-stage renal disease (ESRD) ${ }^{[1]}$. Typically, chronic kidney disease (CKD) develops over 10 years after diagnosis of type 1 diabetes, but may be present at the diagnosis of type 2 diabetes. Middleelderly patients have a relatively high prevalence of type 2 diabetes. Therefore, it's important to concern the early diagnosis of $\mathrm{DN}$ in this segment of population.

Albuminuria is a predictor of future renal dysfunction ${ }^{[2]}$, it is often used as one of the criteria for clinical diagnosis of DN. In the past, urinary albumin excretion rate (UAER) of spot or 24-hour urine were usually tested in the evaluation of albumin loss through kidney ${ }^{[3]}$. However, in recent years, albumin-to-creatinine ratio (UACR) of spot urine, with improved accuracy and convenience, has become more widely accepted in the diagnosis and monitoring of DN. On the other hand, several studies have demonstrated that a considerable portion of diabetic patients with renal dysfunction had no proteinuria ${ }^{[4-6]}$. In our study, UACR of spot urine was tested for the clinical diagnosis of DN, and effective glomerular filtration rate (eGFR) was calculated as well. 
Inflammation plays an important role in the development of diabetes and its complications, including both macro- and micro-vascular diseases ${ }^{[7-11]}$. Neutrophil to lymphocyte ratio (NLR) significantly correlates to CRP and TNF-a levels ${ }^{[12,13]}$, it has been accepted as proportional to the degree of inflammation ${ }^{[14]}$. Previous studies have demonstrated that NLR associated with a worse outcome in patents with diabetes, metabolic syndrome, cancer, stroke and cardiovascular diseases (CVD) ${ }^{[3,15-19]}$, etc.

Several recent studies reported the relationship between NLR and DN. However, most of them used UAER in the diagnosis of $\mathrm{DN}^{[13,20-24]}$, which was less efficient than UACR. More important, an adequate threshold of NLR for the prediction and evaluation of DN remains unclear. This cross-sectional study aimed to investigate the association of NLR and DN in middle-elderly aged patients with type 2 diabetes, and attempted to confirm an optimized cutoff value of NLR in the prediction of DN diagnosis.

\section{Methods}

\section{Subjects}

Inpatients with type 2 diabetes treated in our department were included between July and December 2015. Inclusion criteria were (1) aged from 40 to 80 year-old, (2) diagnosed as type 2 diabetes over 1 year. Exclusion criteria were (1) kidney disease other than DN, use of angiotensin-converting enzyme inhibitors or angiotensin receptor blockers (ACEI/ARB) within one month; (2) acute coronary artery disease or stroke, active inflammation, any kind of cancer; (3) eGFR $<30 \mathrm{ml} / \mathrm{min} \cdot 1.73 \mathrm{~m}^{2}$.

Clinical, anthropometric and laboratory characteristics of included patients were extracted from medical record retrospectively. Data of age, gender, duration of diabetes, history of smoking, past history of CVD, family history of diabetes, blood pressure and body mass index (BMI) were included. Results of blood routine, renal function, lipids, glycated hemoglobin $\mathrm{A} 1 \mathrm{c}(\mathrm{HbA} 1 \mathrm{c})$, fasting and postprandial glucose, insulin and C-peptide were also recorded.

This study was approved by the local ethical committee of the First Hospital of Qinhuangdao (201502A168), and all participants provided written informed consent.

\section{Evaluation of DN and NLR}

Fresh morning spot urine samples were obtained twice on different days for each patients. UACR $(\mathrm{mg} / \mathrm{g})$ was calculated as urine albuminuria $(\mathrm{mg} / \mathrm{dl})$ divided by urine creatinine $(\mathrm{g} / \mathrm{dl})$, and recorded as the average of the twice UACR values. Micro-albuminuria was accepted as $30 \mathrm{mg} / \mathrm{g} \leq \mathrm{UACR}<300 \mathrm{mg} / \mathrm{g}$, macro-albuminuria was defined as UACR $\geq 300 \mathrm{mg} / \mathrm{g}$.

DN was defined as diabetes combined with micro- or macro-albuminuria or eGFR $<60 \mathrm{ml} / \mathrm{min} \cdot 1.73 \mathrm{~m}^{2}$ calculated by Cockcroft-Gault method ${ }^{[25]}$. All included patients were assigned into DN group and non-DN group, accordingly. 
Blood corpuscles were counted for each patient using the LH 780 analyzer (Beckman Coulter Inc, Miami, Florida). NLR was calculated as absolute neutrophil count $(10 * 9 / \mathrm{I})$ divided by lymphocyte count $(10 * 9 / \mathrm{l})$. Similarly, PLR (platelet to lymphocyte ratio) was calculated as platelet count $(10 * 9 / \mathrm{I})$ divided by lymphocyte count $(10 * 9 / I)$.

\section{Statistics}

Statistical analysis was performed using SPSS v21.0 package (IBM, Armonk, NY, USA) and MedCalc software 15.2.2 (Ostend, Belgium). Nominally distributed data were given as mean \pm standard deviation (SD). Non-nominally distributed data were described as median (interquartile range, IQR). Category variables were given as number (percentage). $T$-test was performed to evaluate the difference between independent samples. Fisher's exact test was carried out to analyze the distribution of category variables. Spearman's rho test was performed to evaluate the correlation between independent variables. Binary logistic regression analysis was performed to estimate the contributions of clinical and laboratory variables to DN diagnosis. Canonical discriminant functions and Wilk's lambda test were used to construct the discriminant equation and calculate the predicted probabilities. Receiver operating characteristics (ROC) curve analysis was used to determine the cutoff value of DN diagnosis, the comparisons of AUC were also performed. $P<0.05$ was considered significant.

\section{Results}

\section{Demographic, clinical and laboratory characteristics of patients}

A total of 146 patients with type 2 diabetes were included in this study, comprising 47 patients with DN and 99 patients without DN. There was no significant difference of age and gender distribution between the 2 groups. As compared to non-DN group, DN patients had longer diabetes duration, higher BMI and elevated systolic blood pressure (SBP) levels. Meanwhile, no significant difference was shown of family history of diabetes, past history of CVD and smoking between the 2 groups (table 1 ).

In the view of glucose metabolism, DN patients presented with higher HbA1c and lower C-peptide levels than those of non-DN patients. However, no differences were shown of the plasma glucose and insulin concentrations between the 2 groups, irrespective of fasting or postprandial samples (table 1). As to lipid metabolism, only Lp(a) was demonstrated to be elevated in DN patients as compared to those of non-DN patients. None of the other parameters showed significant difference between the 2 groups (table 1 ), including low-density lipoprotein cholesterol (LDL-C), high-density lipoprotein cholesterol (HDL-c), apolipoprotein A and B (apo-A and Apo-B). Renal function tests showed a significant increase of uric acid, creatinine and UACR in DN patients as compared to those of non-DN patients, while urea nitrogen and eGFR levels showed no difference between the 2 groups (table 1 ).

In the complete blood counts analysis, white blood cell (WBC) and neutrophil counts were demonstrated to be higher in patients with DN than those without DN. However, there was no significant difference of lymphocyte, platelet and monocyte counts between the 2 groups (table 1). Furthermore, when comparing 
the calculated NLR and PLR between the 2 groups, DN patients had a higher NLR level than those of nonDN patients, but PLR showed no difference between the 2 groups (table 1 ).

\section{Analysis of potential confounding factors of NLR}

Since NLR could be easily affected by may other factors, we analyzed the potential confounders included in this study. As a result, weight $(r=0.164, P=0.049), \mathrm{HbA1c}(r=0.219, P=0.010)$, cholesterol $(r=-0.189$, $P=0.023)$, HDL-c $(r=-0.187, P=0.025)$ and apo-A $(r=-0.219, P=0.013)$ significantly associated with NLR levels. However, no correlations were revealed between NLR and other parameters, including age, blood pressure, duration of diabetes, BMI, index of glucose and lipid metabolism except for those mentioned above and variables of renal function $(P>0.05)$. Meanwhile, no significant difference was shown between different groups of gender, with or without past history of CVD, family history of diabetes or smoking history $(P>0.05$, Supplemental table 1$)$.

\section{Predicting ability of NLR for early diagnosis of DN}

To investigate the association of NLR and the 2 main elements (UACR and eGFR) for early diagnosis of DN, Spearman's rho test was performed. As a result, there was a positive correlation of NLR and UACR levels $(r=0.227, P=0.006)$, while no correlation was shown between NLR and eGFR $(r=0.089, P=$ $0.288)$.

In binary logistic regression analysis, duration of diabetes, NLR, SBP and Lp(a) were shown to predict the DN diagnosis independently, after adjusted by multi-variables of age, gender, BMI, smoking history, HbA1c and UA (table 2). This regression model had an overall prediction accuracy of $81.8 \%$, interestingly, the sensitivity and specificity were $62.2 \%$ and $90.5 \%$, respectively. ROC curve analysis indicated that NLR value of 2.04 had a sensitivity of $48.9 \%$ and a specificity of $80.8 \%$ in predicting $D N$, with area under the curve (AUC) of 0.666 [ $P=0.001,95 \% \mathrm{Cl}: 0.584-0.742$, figure $1(\mathrm{a})]$. Notably, when the threshold of NLR was elevated to 2.50 , the specificity for diagnosing DN was $91.9 \%$, even though the sensitivity decreased to $29.8 \%$ at the same point.

Furthermore, we constructed two models using the equations of canonical discriminant functions. Model 1 included the four independent risk factors of diabetes duration, NLR, Lp(a) and SBP. The factor of Lp(a) was excluded in model 2. Statistical characteristics of the two models were shown in supplemental table 2 , and the two equations were listed as below:

\section{Model 1:}

$D=-6.230+0.091 X_{1}+0.612 X_{2}+0.026 X_{3}+0.023 X_{4}$

\section{Model 2:}

$D=-6.718+0.098 X_{1}+0.826 X_{2}+0.030 X_{3}$ 
$\left[\mathrm{X}_{1}=\right.$ diabetes duration (year), $\left.\mathrm{X}_{2}=\mathrm{NLR}, \mathrm{X}_{3}=\operatorname{SBP}(\mathrm{mmHg}), \mathrm{X}_{4}=\mathrm{Lp}(\mathrm{a})(\mathrm{mg} / \mathrm{dl})\right]$

Accordingly, the ROC curve analysis was performed using the predicted probabilities obtained from the discriminant analysis. In model 1 , the predicted probability of 0.29 showed a sensitivity of $76.9 \%$ and a specificity of $75.3 \%$, with the AUC of $0.819[P<0.001,95 \% \mathrm{Cl}$ : $0.741-0.881$, figure $1(\mathrm{~b})]$. In model 2 , the predicted probability of 0.47 had a sensitivity of $74.4 \%$ and a specificity of $78.7 \%$, with the AUC of 0.817 $[P<0.001,95 \% \mathrm{Cl}: 0.739-0.879$, figure $1(\mathrm{c})]$. In a word, when the calculated $D>0.29$ and 0.47 , the individual patient could be classified as a DN patient according to model 1 and model 2, respectively. When comparing different ROC curves, AUC of both model 1 and model 2 showed significant improvements as compared to that of NLR $(P<0.05)$. However, there was no significant difference of AUC between model 1 and model 2 (table 3 ).

\section{Discussion}

With rapidly increased prevalence, diabetes and its chronic complications have drawn more concerns of people ${ }^{[26]}$. DN is a common complication of diabetes, it usually starts from glomerular damage indicated by micro-albuminuria ${ }^{[2]}$. Recently, American Diabetes Association (ADA) recommended to cancel the statement of "micro-" or "macro-albuminuria" in the consideration of the continuousness of disease. On the other hand, tubulo-interstitial injury is also responsible for increased protein filtration and loss of renal function ${ }^{[26]}$. Therefore, in this study, we defined DN as UACR $>30 \mathrm{mg} / \mathrm{g}$ or eGFR $<60 \mathrm{ml} / \mathrm{min} \cdot 1.73 \mathrm{~m}^{2}$ or both, but those with eGFR $<30 \mathrm{ml} / \mathrm{min} \cdot 1.73 \mathrm{~m}^{2}$ was excluded for the purpose of early diagnosis.

Inflammation plays an important role in the development and progression of DN. NLR could be used as a marker of systemic inflammation. As previously reported, diabetic patients were prone to have higher NLR levels than those of healthy volunteers $[1.93 \operatorname{IQR}(1.43,2.68) \text { vs } 1.61 \operatorname{IQR}(1.31,2.16), P<0.001]^{[26]}$. A longitudinal study reported that, after 3-year follow-up of diabetic patients, the lowest NLR tertile included fewer patients $(2.7 \%)$ of worsening renal functions than those of the middle and the highest NLR tertiles ${ }^{[20]}$. Based on the results of our study, NLR positively correlated to UACR in patients with type 2 diabetes, DN patients had higher NLR levels than those of non-DN patients $(2.21 \pm 1.05$ vs $1.67 \pm 0.71$, $P=0.002$ ), corresponding to the results of previous studies ${ }^{[13,21,22,24,27,28]}$. Some of the studies didn't provide the values of NLR in DN and non-DN groups ${ }^{[3,21]}$. Other clinical researches gave the mean values of NLR ranged from 1.56 to $2.20,1.96$ to 2.60 and 2.03 to 3.60 in diabetic patients with normo-, microand macro-alubuminuria, respectively ${ }^{[13,22,24,29]}$. Apparently, there were overlaps among different groups, which further supported DN to be a kind of continuously progressed disease.

Moreover, NLR independently predicted DN diagnosis after adjusted by multi-variables, based on the results of both our study and previous studies ${ }^{[21,22,30]}$. However, an adequate cutoff value of NLR had never been clearly elucidated in the past. A recently published study of meta-analysis ever focused on this point of view. Regretfully, the results only provided a standardized mean difference (SMD) value of NLR $(S M D=0.63,95 \% \mathrm{Cl} \text { : } 0.43-0.83, P<0.001)^{[31]}$, which could scarcely help clinical doctors to make any 
decisions. ROC curve analysis had also been preformed by some of the studies. Akbas et al ${ }^{[26]}$ reported a NLR cutoff value of 1.7 in predicting albuminuria of diabetes, with a sensitivity of $61.8 \%$ and a specificity of $70.5 \%$ (AUC $0.660,95 \% \mathrm{Cl} 0.590-0.725, P=0.0001$ ). This result is similar to the ROC analysis of our study that NLR cutoff value of 2.04 had a sensitivity of $48.9 \%$, a specificity of $80.8 \%$, and AUC of 0.666 . However, these results also revealed that NLR, as a single predictor, had a moderate efficiency in predicting $D N$, let alone the fact that NLR value of 1.7 and 2.04 could barely separate DN from diabetic patients without DN (with mean NLR value from 1.56 to $2.20^{[13,22,24,29]}$ ). A more efficient but simple model was necessary.

Based on the logistic regression analysis, we selected 4 independent factors [duration of diabetes, NLR, SBP and Lp(a)] as variables for the construction of discriminant equation in predicting DN. Therefore, model 1 was established. Using this model, the AUC was elevated to 0.819 with a sensitivity of $76.9 \%$ and a specificity of $75.3 \%$. However, Lp(a) might not be easily acquired in some cases. Accordingly, model 2 was established excluding the factor of $L p(a)$, with only a slight loss of AUC (0.817) compared to model 1 (0.819), but an obvious improvement of sensitivity (74.4\%) compared to NLR alone (48.9\%).

In clinical practice, invasive kidney biopsy was rarely operated in the diagnosis of $D N$, repeated blood drawing and expensive urine test were usually opposed too. The medical expense was another concern. Routine blood test remained more easier to be accepted by most of the patients. In this situation, when the lab data of creatine and UACR were difficult to obtain, we recommended clinical practitioners to use model 1 or model 2 for quick glimpses at DN prediction. Because they were user-friendly and easily to be calculated, with an acceptable predictive accuracy, and might be easily stored as an excel document in the office computer. Especially the model 2, which need only 2 additional clinical parameters of SBP and diabetes duration, except for NLR value.

In addition, according to our study, NLR value of 2.50 with a high specificity $(91.9 \%)$ is another userfriendly tool to identify the patients at high risk of DN, even if it had a fairly low sensitivity of $29.8 \%$. It would be useful to remind doctors to pay attention to the high probability of $\mathrm{DN}$, and persuade this part of patients to accept necessary blood or urine tests further. For another important reason, as clinical doctors, we deeply understood that there were too many data need to be remember in daily work, and too many trivial things to distract our attentions, so a very simple but efficient tool would be more fascinated. Above all, even the discriminant analysis provided a method of better overall accuracy in predicting DN, we still strongly recommended the NLR threshold of 2.50 as the clinical use to locate the patients at high risk of DN, irrespective of the low sensitivity. Because it's pretty simple and easy to be remembered. With this tool alone, we might specifically determine around $1 / 3$ patients with $D N$ only, but without it, we may lose all of them.

With regards to the inner relationship between NLR and DN, in a hypothesis, excessive nutrients could activate the pancreatic islets, liver, adipose and muscle tissues to release chemokine and cytokines, such as tumor necrosis factor-a (TNF-a), interleukin-1 (IL-1) and interleukin-8 (IL-8, also called CXCL-8). They could help to recruit immune cells and promote inflammation ${ }^{[8]}$. In particular, IL-8 was the specific 
chemokine of neutrophils. When binding to CXCR-1 and CXCR-2 ${ }^{[32]}$, IL-8 could induce chemotaxis, migration, aggregation and activation of neutrophils, and participate in tissue injury and repair. A previous animal study had shown that IL-8 antagonist could reduce renal volume and UACR level, improve creatinine clearance in male mice with diabetes, attenuate high glucose induced mesangial injury, and inhibit JAK2/STAT3 and ERK1/2 pathways at molecular level[ ${ }^{[33]}$. In vivo study had also indicated an elevated level of urinary CXCL-8 of DN patients as compared to those of control[26].

Our study had several limitations. First, this was a retrospective study with relatively small sample size, leading to the restricted generalization of the results. Validation of some large-scale studies remained necessary. Second, a validation cohort is absent, even though the results of our study were quite close to those of previously reported. We also look forward other researchers to verifying our results using their reported data set. Third, the factors impact on NLR were not completely expelled, like the most frequent complications of hypertension and dyslipidemia. Of course, we've already tried to exclude their influences by performing multi-variable regression analysis. On the other hand, they were truly existing in the real world, and probably shared a common mechanism with diabetes and DN.

\section{Conclusions}

Two models of user-friendly equations were constructed for early prediction of $\mathrm{DN}$, which could be easily calculated and stored in office computer. NLR threshold of 2.50 is strongly recommended in clinical use to identify the patients at high risk of DN, for its high specificity and remarkable convenience.

\section{Abbreviations}

NLR, neutrophil to lymphocyte ratio;

DN, diabetic nephropathy;

eGFR, effective glomerular filtration rate;

SBP, systolic blood pressure;

$L p(a)$, lipoprotein a;

AUC, area under the curve.

\section{Declarations}

Ethics approval and consent to participate

This study was approved by the Committee of Ethics in Research of the First Hospital of Qinhuangdao (20201311). All participants provided their written informed consent to participate in this study, after approval of the process by the ethics committee. 
Consent for publication

Not applicable.

Availability of data and materials

The dataset used and analysed during the current study are available from the corresponding author on reasonable request.

Competing interests

The authors declare that they have no conflict of interest.

Funding

This study was nominally supported by the Medical Science Research Project of Hebei Province [Grant number: 20201311] without financial support.

Authors' contributions

YMT collected, analyzed data and drafted the manuscript. TL, HYG and RW collected clinical data. CMM made substantial contributions to acquisition of data. RW and FZY revised it critically for important intellectual content.

Acknowledgements

We thank Dr.JD Liu and Dr.D Zhu for the assistance of clinical data collection.

\section{References}

1. Standards of Medical Care in Diabetes-2015: Summary of Revisions. Diabetes Care 2014, 38:S4-S4.

2. Retnakaran R, Cull CA, Thorne KI, Adler Al, Holman RR: Risk factors for renal dysfunction in type 2 diabetes: U.K. Prospective Diabetes Study 74. Diabetes 2006, 55(6):1832-1839.

3. Kawamoto RA-O, Ninomiya D, Kikuchi A, Akase T, Kasai Y, Kusunoki T, Ohtsuka N, Kumagi T: Association of neutrophil-to-lymphocyte ratio with early renal dysfunction and albuminuria among diabetic patients. (1573-2584 (Electronic)).

4. Kramer HJ, Nguyen QD, Curhan G, Hsu C-y: Renal Insufficiency in the Absence of Albuminuria and Retinopathy Among Adults With Type 2 Diabetes Mellitus. JAMA 2003, 289(24):3273-3277.

5. An JH, Cho YM, Yu HG, Jang HC, Park KS, Kim SY, Lee HK: The Clinical Characteristics of Normoalbuminuric Renal Insufficiency in Korean Type 2 Diabetic Patients: A Possible Early Stage Renal Complication. J Korean Med Sci 2009, 24(Suppl 1):S75-S81. 
6. Zachwieja J, Soltysiak J, Fichna P, Lipkowska K, Stankiewicz W, Skowronska B, Kroll P, Lewandowska-Stachowiak M: Normal-range albuminuria does not exclude nephropathy in diabetic children. Pediatric Nephrology 2010, 25(8):1445-1451.

7. Navarro JF, Mora C: Role of inflammation in diabetic complications. Nephrol Dial Transplant 2005, 20(12):2601-2604.

8. Donath MY, Shoelson SE: Type 2 diabetes as an inflammatory disease. Nat Rev Immuno/ 2011, 11(2):98-107.

9. Ozturk ZA, Kuyumcu ME, Yesil Y, Savas E, Yildiz H, Kepekci Y, Ariogul S: Is there a link between neutrophil-lymphocyte ratio and microvascular complications in geriatric diabetic patients? $J$ Endocrinol Invest 2013, 36(8):593-599.

10. Xu T, Weng Z, Pei C, Yu S, Chen Y, Guo W, Wang X, Luo P, Sun J: The relationship between neutrophilto-lymphocyte ratio and diabetic peripheral neuropathy in Type 2 diabetes mellitus. Medicine (Baltimore) 2017, 96(45):e8289.

11. Demirdal T, Sen P: The significance of neutrophil-lymphocyte ratio, platelet-lymphocyte ratio and lymphocyte-monocyte ratio in predicting peripheral arterial disease, peripheral neuropathy, osteomyelitis and amputation in diabetic foot infection. Diabetes research and clinical practice 2018, 144:118-125.

12. Lorenzo C, Hanley AJ, Haffner SM: Differential white cell count and incident type 2 diabetes: the Insulin Resistance Atherosclerosis Study. Diabetologia 2014, 57(1):83-92.

13. Kahraman C, Kahraman NK, Aras B, Cosgun S, Gulcan E: The relationship between neutrophil-tolymphocyte ratio and albuminuria in type 2 diabetic patients: a pilot study. Arch Med Sci 2016, 12(3):571-575.

14. Imtiaz F, Shafique K, Mirza SS, Ayoob Z, Vart P, Rao S: Neutrophil lymphocyte ratio as a measure of systemic inflammation in prevalent chronic diseases in Asian population. Int Arch Med 2012, 5(1):2.

15. Davis JL, Moutinho V, Jr., Panageas KS, Coit DG: A peripheral blood biomarker estimates probability of survival: the neutrophil-lymphocyte ratio in noncancer patients. Biomark Med 2016, 10(9):953-957.

16. Kim do Y, Choi SW, Han SW, Youn JC, Ryu KH: OS 01-03 NEUTROPHIL TO LYMPHOCYTE RATIO IS CLOSELY RELATED WITH BLOOD PRESSURE LEVEL IN HYPERTENSIVE INDIVIDUALS WITHOUT CARDIOVASCULAR DISEASES; DATA FROM THE KOREAN REGISTRY OF TARGET ORGAN DAMAGES IN HYPERTENSION (KorHR). J Hypertens 2016, 34 Suppl 1:e44-45.

17. Min K, Kwon S, Cho SY, Choi WJ, Park SU, Jung WS, Moon SK, Park JM, Ko CN, Cho KH: Atrial Fibrillation is Strongly Associated With the Neutrophil to Lymphocyte Ratio in Acute Ischemic Stroke Patients: A Retrospective Study. J Clin Lab Anal 2016.

18. Surendar J, Indulekha K, Mohan V, Pradeepa R: Association of neutrophil-lymphocyte ratio with metabolic syndrome and its components in Asian Indians (CURES-143). J Diabetes Complications 2016, 30(8):1525-1529.

19. Hussain M, Babar MZM, Akhtar L, Hussain MS: Neutrophil lymphocyte ratio (NLR): A well assessment tool of glycemic control in type 2 diabetic patients. Pak J Med Sci 2017, 33(6):1366- 
1370.

20. Azab B, Daoud J, Naeem FB, Nasr R, Ross J, Ghimire P, Siddiqui A, Azzi N, Rihana N, Abdallah M et al: Neutrophil-to-lymphocyte ratio as a predictor of worsening renal function in diabetic patients (3-year follow-up study). Ren Fail 2012, 34(5):571-576.

21. Afsar B: The relationship between neutrophil lymphocyte ratio with urinary protein and albumin excretion in newly diagnosed patients with type 2 diabetes. Am J Med Sci 2014, 347(3):217-220.

22. Akbas EM, Demirtas L, Ozcicek A, Timuroglu A, Bakirci EM, Hamur H, Ozcicek F, Turkmen K: Association of epicardial adipose tissue, neutrophil-to-lymphocyte ratio and platelet-to-lymphocyte ratio with diabetic nephropathy. Int J Clin Exp Med 2014, 7(7):1794-1801.

23. Shiny A, Bibin YS, Shanthirani CS, Regin BS, Anjana RM, Balasubramanyam M, Jebarani S, Mohan V: Association of neutrophil-lymphocyte ratio with glucose intolerance: an indicator of systemic inflammation in patients with type 2 diabetes. Diabetes Technol Ther 2014, 16(8):524-530.

24. Huang W, Huang J, Liu Q, Lin F, He Z, Zeng Z, He L: Neutrophil-lymphocyte ratio is a reliable predictive marker for early-stage diabetic nephropathy. Clin Endocrinol (Oxf) 2015, 82(2):229-233.

25. Cockcroft Dw Fau - Gault MH, Gault MH: Prediction of creatinine clearance from serum creatinine. (1660-8151 (Print)).

26. !!! INVALID CITATION !!!

27. Yilmaz H, Ucan B, Sayki M, Unsal I, Sahin M, Ozbek M, Delibasi T: Usefulness of the neutrophil-tolymphocyte ratio to prediction of type 2 diabetes mellitus in morbid obesity. Diabetes Metab Syndr 2014.

28. Chittawar S, Dutta D, Qureshi Z, Surana V, Khandare S, Dubey TN: Neutrophil-lymphocyte Ratio is a Novel Reliable Predictor of Nephropathy, Retinopathy, and Coronary Artery Disease in Indians with Type-2 Diabetes. Indian J Endocrinol Metab 2017, 21(6):864-870.

29. Turkmen S, Dogdu O, Tekin K, Kucukdurmaz Z, Cagliyan CE, Sarikaya S, Yucel H, Karapinar H, Ozkan $\mathrm{B}$, Uysal OK et al: The relationship between neutrophil/lymphocyte ratio and the TIMI flow grade in patients with STEMI undergoing primary PCI. Eur Rev Med Pharmacol Sci 2013, 17(16):2185-2189.

30. Ciray H, Aksoy AH, Ulu N, Cizmecioglu A, Gaipov A, Solak Y: Nephropathy, but not Angiographically Proven Retinopathy, is Associated with Neutrophil to Lymphocyte Ratio in Patients with Type 2 Diabetes. (1439-3646 (Electronic)).

31. Liu J, Liu X, Li Y, Quan J, Wei S, An S, Yang R, Liu J: The association of neutrophil to lymphocyte ratio, mean platelet volume, and platelet distribution width with diabetic retinopathy and nephropathy: a meta-analysis. LID - BSR20180172 [pii] LID - 10.1042/BSR20180172 [doi]. (15734935 (Electronic)).

32. Tanaka K, Yoshitomi T, Hirahara K: Elucidation of Distinct Roles of Guinea Pig CXCR1 and CXCR2 in Neutrophil Migration toward IL-8 and GROalpha by Specific Antibodies. Biol Pharm Bull 2017, 40(5):729-732.

33. Cui S, Zhu Y, Du J, Khan MN, Wang B, Wei J, Cheng JW, Gordon JR, Mu Y, Li F: CXCL8 Antagonist Improves Diabetic Nephropathy in Male Mice With Diabetes and Attenuates High Glucose-Induced 
Mesangial Injury. Endocrinology 2017, 158(6):1671-1684.

\section{Tables}

Table 1. Demographic, clinical and laboratory characteristics of the patients in DN and non-DN groups. 


\begin{tabular}{|c|c|c|c|}
\hline & DN group & non-DN group & $P$-value \\
\hline gender ( $n$ of male/female) & $26 / 21$ & $50 / 49$ & 0.600 \\
\hline age (year) & $61.21 \pm 9.99$ & $58.25 \pm 8.94$ & 0.074 \\
\hline diabetes duration (year) & $13(8,18)$ & $9(6,13)$ & $0.007 *$ \\
\hline family history of Diabetes (\% of yes) & 55.3 & 44.4 & 0.287 \\
\hline past history of CVD (\% of yes) & 29.8 & 27.3 & 0.844 \\
\hline Smoking (\% of yes) & 38.3 & 27.3 & 0.186 \\
\hline weight $(\mathrm{kg})$ & $77.11 \pm 17.17$ & $72.43 \pm 11.70$ & 0.057 \\
\hline body mass index $(\mathrm{kg} / \mathrm{m} 2)$ & $27.90 \pm 5.85$ & $26.04 \pm 3.25$ & $0.049^{*}$ \\
\hline SBP (mmHg) & $142.26 \pm 16.43$ & $143.19 \pm 15.81$ & $0.005^{*}$ \\
\hline $\mathrm{DBP}(\mathrm{mmHg})$ & $82.00 \pm 13.04$ & $83.03 \pm 9.63$ & 0.631 \\
\hline HbA1c (\%) & $9.29 \pm 1.54$ & $8.56 \pm 1.90$ & $0.027 *$ \\
\hline FBG $(\mathrm{mmol} / \mathrm{l})$ & $9.41 \pm 3.51$ & $9.40 \pm 3.23$ & 0.982 \\
\hline PBG2h (mmol/l) & $14.59 \pm 4.07$ & $15.34 \pm 5.19$ & 0.436 \\
\hline $\mathrm{FBI} \square \mathrm{uIU} / \mathrm{ml} \square$ & $10.12 \pm 7.13$ & $10.01 \pm 4.01$ & 0.947 \\
\hline $\mathrm{PBI} 2 \mathrm{~h} \rrbracket \mathrm{uIU} / \mathrm{ml} \square$ & $29.65 \pm 16.91$ & $33.03 \pm 32.69$ & 0.725 \\
\hline $\mathrm{FBCP} \square \mathrm{ng} / \mathrm{ml} \square$ & $1.07 \pm 0.59$ & $1.45 \pm 0.69$ & $0.012^{*}$ \\
\hline $\mathrm{PBCP} 2 \mathrm{~h} \rrbracket \mathrm{ng} / \mathrm{ml} \square$ & $2.43 \pm 1.46$ & $3.36 \pm 2.12$ & $0.034^{*}$ \\
\hline triglyceride (mmol/l) & $2.17 \pm 2.05$ & $2.02 \pm 1.35$ & 0.588 \\
\hline cholesterol (mmol/l) & $5.22 \pm 2.17$ & $4.78 \pm 1.01$ & 0.188 \\
\hline LDL-c (mmol/l) & $3.29 \pm 1.41$ & $3.12 \pm 0.85$ & 0.437 \\
\hline HDL-c $(\mathrm{mmol} / \mathrm{l})$ & $1.25 \pm 0.48$ & $1.10 \pm 0.33$ & 0.064 \\
\hline Apo-A (g/l) & $1.09 \pm 0.23$ & $1.18 \pm 1.07$ & 0.589 \\
\hline Apo-B (g/l) & $1.01 \pm 0.42$ & $0.94 \pm 0.21$ & 0.321 \\
\hline lipoprotein a (mg/dl) & $31.25 \pm 25.67$ & $18.83 \pm 14.14$ & $0.007 *$ \\
\hline uric acid $(\mu \mathrm{mol} / \mathrm{L})$ & $331.22 \pm 96.10$ & $298.59 \pm 75.65$ & $0.029 *$ \\
\hline urea nitrogen (mmol/l) & $6.38 \pm 1.84$ & $5.84 \pm 1.62$ & 0.075 \\
\hline creatinine $(\mu \mathrm{mol} / \mathrm{L})$ & $69.39 \pm 25.10$ & $56.21 \pm 13.30$ & $0.001 *$ \\
\hline WBC count $(10 * 9 / 1)$ & $7.09 \pm 1.65$ & $6.44 \pm 1.72$ & $0.033^{*}$ \\
\hline neutrophil count $(10 * 9 / 1)$ & $4.24 \pm 1.48$ & $3.56 \pm 1.32$ & $0.006^{*}$ \\
\hline lymophocyte count $(10 * 9 / 1)$ & $2.09 \pm 0.62$ & $2.22 \pm 0.72$ & 0.276 \\
\hline platelet count $(10 * 9 / 1)$ & $245.52 \pm 81.76$ & $241.41 \pm 238.36$ & 0.910 \\
\hline monocyte count $(10 * 9 / 1)$ & $0.54 \pm 0.14$ & $0.50 \pm 0.21$ & 0.256 \\
\hline NLR & $2.21 \pm 1.05$ & $1.67 \pm 0.71$ & $0.002 *$ \\
\hline PLR & $126.77 \pm 56.75$ & $139.77 \pm 137.61$ & 0.738 \\
\hline UACR (mg/g) & $21.91 \pm 24.97$ & $0.94 \pm 075$ & $<0.001^{*}$ \\
\hline eGFR $\left(\mathrm{ml} / \mathrm{min} \cdot 1.73 \mathrm{~m}^{2}\right)$ & $115.65 \pm 56.22$ & $123.92 \pm 34.05$ & 0.279 \\
\hline
\end{tabular}


$* P<0.05$

DN, diabetic nephropathy; CVD, cardiovascular disease; SBP, systolic blood pressure; DBP, diastolic blood pressure; HbA1c, glycated hemoglobin A1c; FBG, fast blood glucose; PBG2h, postprandial blood pressure of 2 hour; FBI, fast blood insulin; PBI2h, postprandial blood insulin of 2 hour; FBCP, fast blood c-peptide; PBCP2h, postprandial blood c-peptide of 2 hour; LDL-c, low-density lipoprotein cholesterol; HDL-c, high-density lipoprotein cholesterol; Apo-A, apo-lipoprotein A; Apo-B, apo-lipoprotein B; WBC, white blood cell; NLR, neutrophil to lymphocyte ratio; PLR, platelet to lymphocyte ratio; UACR, urine albumin to creatinine ratio; effective glomerular filtration rate.

Table 2. Binary logistic regression analysis of DN with adjustments of multi-variables.

\begin{tabular}{|c|c|c|c|}
\hline Variables & Wald & $P$-value & $O R \square 95 \% \mathrm{CI} \square$ \\
\hline Diabetes duration & 12.436 & $<0.001 *$ & $1.164 \llbracket 1.070-1.266 \square$ \\
\hline NLR & 6.288 & $0.012 *$ & $2.159 \llbracket 1.183-3.939 \square$ \\
\hline SBP & 5.189 & $0.023^{*}$ & $1.039 \square 1.005-1.073 \square$ \\
\hline $\operatorname{Lp}(\mathrm{a})$ & 4.528 & $0.033^{*}$ & $1.027 \square 1.002-1.053 \square$ \\
\hline Age & 0.144 & 0.704 & $0.989 \llbracket 0.937-1.045 \square$ \\
\hline Gender & 1.729 & 0.189 & $2.170 \square 0.684-6.885 \square$ \\
\hline BMI & 2.246 & 0.134 & $1.103 \llbracket 0.970-1.253 \square$ \\
\hline smoking history & 0.271 & 0.603 & $1.353 \llbracket 0.434-4.221 \square$ \\
\hline HbA1c & 1.244 & 0.265 & $1.170 \square 0.888-1.542 \square$ \\
\hline UA & 1.139 & 0.286 & $1.004 \llbracket 0.997-1.010 \square$ \\
\hline Constant & 15.246 & $<0.001 *$ & \\
\hline
\end{tabular}

$* P<0.05$.

DN, diabetic nephropathy; NLR, neutrophil to lymphocyte ratio; SBP, systolic blood pressure; Lp(a), lipoprotein-a; BMI, body mass index; HbA1c, glycated hemoglobin A1c; UA, uric acid.

Table 3. Comparisons of area under the curve (AUC) for DN prediction.

\begin{tabular}{llll}
\hline & NLR Model1 & NLR Model 2 & Model 1 Model 2 \\
\hline Difference between the areas & 0.169 & 0.166 & 0.00216 \\
Standard error & 0.0587 & 0.0604 & 0.00417 \\
$95 \%$ Confidence interval & $0.0535-0.284$ & $0.0480-0.285$ & $-0.00600-0.0103$ \\
$Z$ statistics & 2.872 & 2.754 & 0.519 \\
$P$ value & $0.0041^{*}$ & $0.0059^{*}$ & 0.6039 \\
\hline
\end{tabular}

$* P<0.05$

NLR, neutrophil to lymphocyte ratio. 
Supplemental table 1. NLR values in different groups of potential confounders.

\begin{tabular}{llll}
\hline & Yes & no & $P$-value \\
\hline Gender of female & $1.87 \pm 1.00$ & $1.83 \pm 0.74$ & 0.778 \\
past history of DM & $1.81 \pm 0.78$ & $1.88 \pm 0.95$ & 0.600 \\
past history of CVD & $1.96 \pm 0.98$ & $1.80 \pm 0.82$ & 0.313 \\
smoking history & $1.78 \pm 0.77$ & $1.88 \pm 0.92$ & 0.517 \\
\hline
\end{tabular}

DM, diabetes mellitus; CVD, cardiovascular disease.

Supplemental table 2. Statistical characteristics of the 2 constructed discriminant equations.

\begin{tabular}{lll}
\hline & Model 1 & Model 2 \\
\hline Wilk's lambda value & 0.746 & 0.794 \\
$\chi^{2}$ statistics & 36.381 & 32.958 \\
degree of freedom & 4 & 3 \\
$P$-value & $<0.001^{*}$ & $<0.001^{*}$ \\
centroid of DN group & 0.875 & 0.735 \\
centroid of non-DN group & -0.384 & -0.349 \\
\hline
\end{tabular}

$* P<0.05$.

\section{Figures}




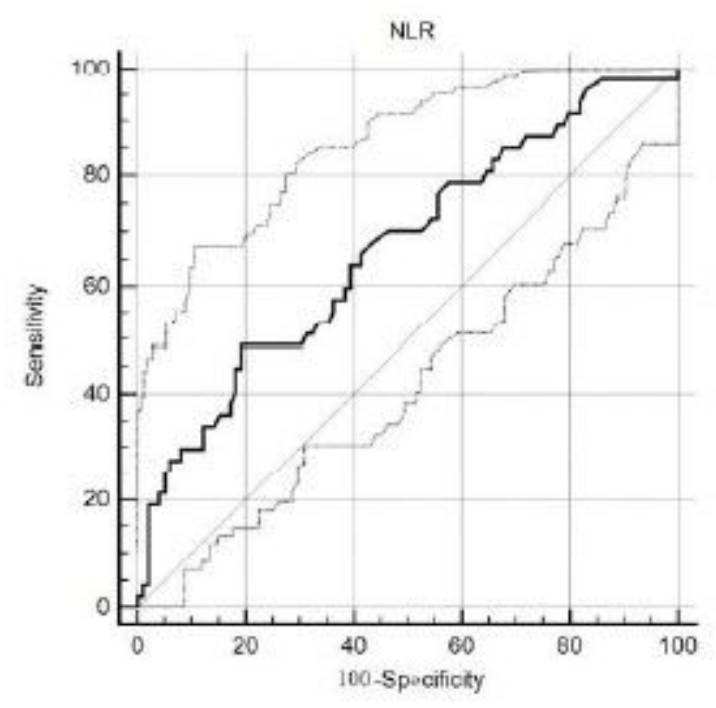

a

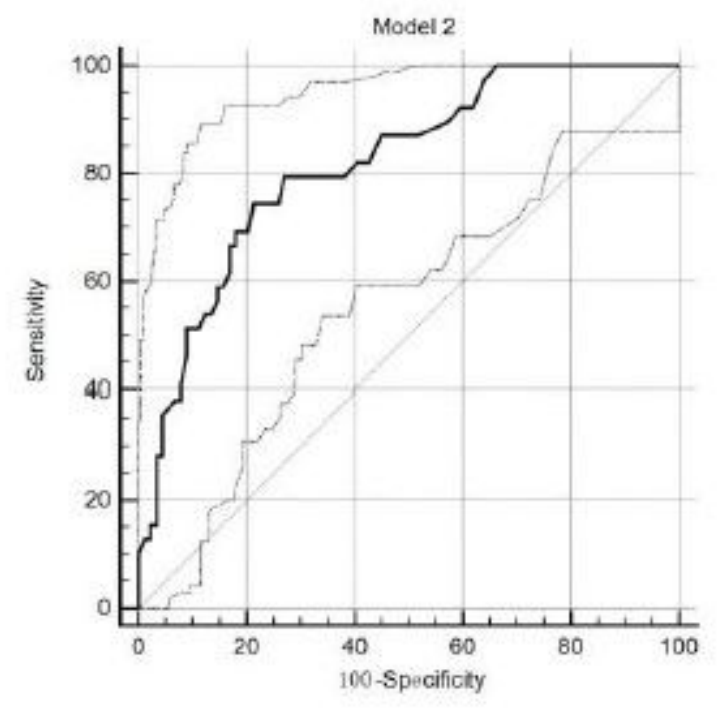

C

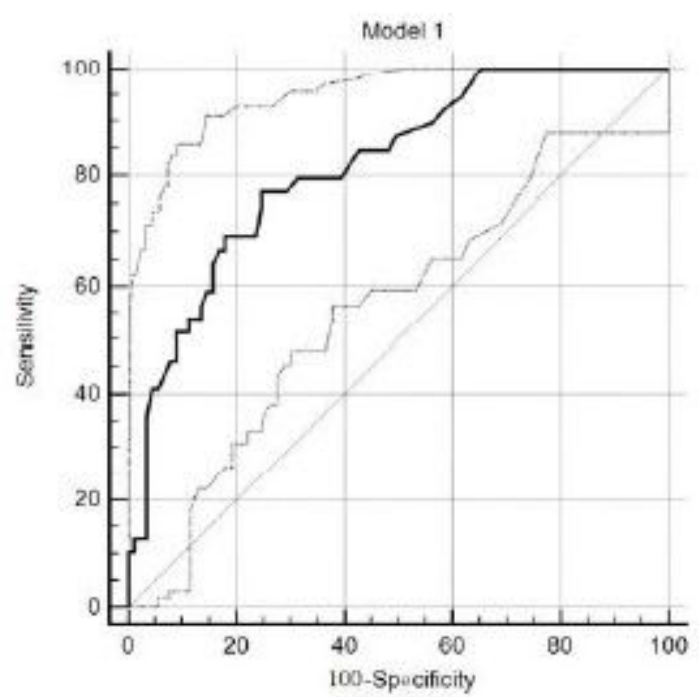

b

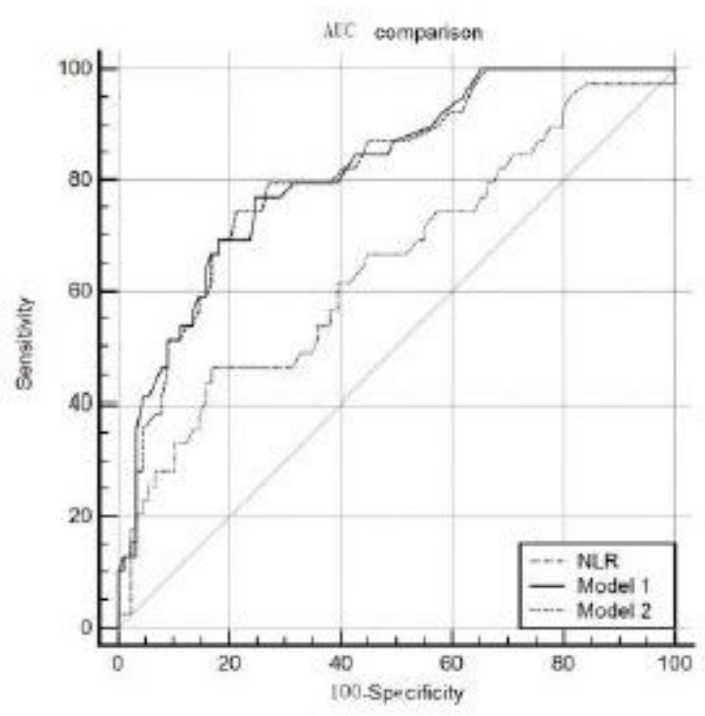

d

\section{Figure 1}

(Receiver operating characteristics (ROC curve analysis indicated that NLR value of 2.04 had a sensitivity of $48.9 \%$ and a specificity of 80 in predicting DN, with area under the curve ( of $0.666 \mathrm{P} 0.001,95 \% \mathrm{Cl}$ : 0.5840 .742 ). ( Model 1 had a sensitivity of $76.9 \%$ and a specificity of $75.3 \%$, with the AUC of $0.819 \mathrm{P}$ $0.001,95 \% \mathrm{Cl}: 0.7410 .881$ ), using the predicted probabilities of d discriminant analysis. (Model 2 had a sensitivity of $74.4 \%$ and a specificity of $78.7 \%$, with the AUC of $0.817 \mathrm{P} 0.001,95 \% \mathrm{Cl}: 0.7390 .879$ ), using the predicted probabilities of discriminant analysis. ( Comparisons of AUC for different ROC curves in $p$ predicting DN diagnosis. 